\title{
BINOMIAL ARITHMETICAL RANK OF EDGE IDEALS OF FORESTS
}

\author{
KYOUKO KIMURA AND NAOKI TERAI
}

(Communicated by Irena Peeva)

\begin{abstract}
We prove that the binomial arithmetical rank of the edge ideal of a forest coincides with its big height.
\end{abstract}

\section{INTRODUCTION}

Let $S=K\left[x_{1}, x_{2}, \ldots, x_{n}\right]$ be a polynomial ring over a field $K$ and $I$ a squarefree monomial ideal of $S$. The arithmetical rank of $I$ is defined by the minimum number $r$ of elements $a_{1}, a_{2}, \ldots, a_{r} \in S$ which generate $I$ up to radical, that is, $\sqrt{\left(a_{1}, a_{2}, \ldots, a_{r}\right)}=I$ holds. We denote it by ara $I$. The binomial arithmetical rank of $I$ is defined by the minimum number $r$ of binomials or monomials $a_{1}, a_{2}, \ldots, a_{r} \in S$ which generate $I$ up to radical. We denote it by biara $I$. By Lyubeznik [12] we know that

$$
\operatorname{pd} S / I \leq \operatorname{ara} I \leq \operatorname{biara} I,
$$

where pd $S / I$ denotes the projective dimension of $S / I$. We are interested in the problem when the equality ara $I=\mathrm{pd} S / I$ holds. It is proved for some classes of squarefree monomial ideals; see, e.g., 2, 3, 4, 5, 6, 1, 8, 9, 10, 11, 13, 14, 16.

In this paper, a graph is assumed to be finite and simple. Denote by $G=$ $(V(G), E(G))$ the graph with the vertex set $V(G)$ and the edge set $E(G)$. We consider the polynomial ring $K[V(G)]$ whose variables are $x_{v}, v \in V(G)$. The ideal of $K[V(G)]$ generated by the quadratic squarefree monomials $x_{u} x_{v},\{u, v\} \in E(G)$ is called the edge ideal of $G$ and denoted by $I(G)$. We work on the above problem for the edge ideals of forests, graphs with no cycle. This was first discussed by Barile [1] and settled for special forests $G$.

In this paper, we compare the binomial arithmetical rank with the big height instead of the projective dimension. Here the big height of a squarefree monomial ideal $I$, denoted by bight $I$ is the maximum height of the minimal prime ideals of $I$. Note that the inequality bight $I \leq \operatorname{pd} S / I$ always holds. Precisely, we prove the following theorem:

Theorem 1.1. Let $G$ be a forest. Then

$$
\text { bight } I(G)=\operatorname{pd} K[V(G)] / I(G)=\operatorname{ara} I(G)=\operatorname{biara} I(G) .
$$

Received by the editors June 27, 2011 and, in revised form, September 26, 2011.

2010 Mathematics Subject Classification. Primary 13F55, 05 C05.

Key words and phrases. Binomial arithmetical rank, primitive tree, tree-like system, edge ideal. 
To obtain Theorem 1.1 through combinatorial interpretations of the big height and binomial arithmetical rank of the edge ideal of a tree, we prove a purely graphtheoretic theorem, which shows an existence of a certain decomposition of a tree. See Theorem 3.2

\section{TREe-LiKe Systems AND PRIMitive TREeS}

In this section we define some notions on a graph which have connections with the binomial arithmetical rank of an edge ideal.

First we introduce a notion of a tree-like system.

Definition 2.1. Let $G$ be a graph. We call the sequence $\Sigma: \mathcal{T}_{1}, \mathcal{T}_{2}, \ldots, \mathcal{T}_{r}$ of nonempty subsets of $E(G)$ with $\# \mathcal{T}_{1}=1, \# \mathcal{T}_{i} \leq 2, i=2,3, \ldots, r$ a tree-like system of $G$ with length $r$ if the following two conditions are satisfied:

(1) The edge set $E(G)$ of $G$ is the disjoint union of $\mathcal{T}_{1}, \mathcal{T}_{2}, \ldots, \mathcal{T}_{r}$.

(2) For each $i$ with $\# \mathcal{T}_{i}=2$, there exist an integer $j<i$ and two vertices $u, v$ each of which is contained in an edge in $\mathcal{T}_{i}$ such that $\{u, v\} \in \mathcal{T}_{j}$.

Remark 2.2. Barile [1, Definition 1, p. 4681] first introduced a tree-like system, which has little difference from ours.

A fundamental technique in the study of the arithmetical rank is the following lemma due to Schmitt and Vogel [15.

Lemma 2.3 (Schmitt and Vogel [15, Lemma, p. 249]). Let $S$ be a ring and $P_{1}, P_{2}, \ldots, P_{r}$ finite subsets of $S$. Assume that $P_{1}, P_{2}, \ldots, P_{r}$ satisfy the following two conditions:

(1) $\# P_{1}=1$.

(2) For all $i>1$ and $a, a^{\prime \prime} \in P_{i}, a \neq a^{\prime \prime}$, there exists $j<i$ and $a^{\prime} \in P_{j}$ such that $a a^{\prime \prime} \in\left(a^{\prime}\right)$.

Let $I$ be an ideal generated by $P_{1}, P_{2}, \ldots, P_{r}$. We set

$$
q_{i}=\sum_{a \in P_{i}} a, \quad i=1,2, \ldots, r .
$$

Then $q_{1}, q_{2}, \ldots, q_{r}$ generate $I$ up to radical.

We can apply Lemma 2.3 to have the following proposition.

Proposition 2.4. If a graph $G$ has a tree-like system of length $r$, then

$$
\text { biara } I(G) \leq r .
$$

A tree is defined to be a connected forest. Next, we introduce a notion of a primitive tree and define for a tree $T$ the number $b(T)$, which is a kind of combinatorial interpretation of the binomial arithmetical rank of an edge ideal.

Definition 2.5. We say that a tree $T$ is primitive if it is obtained by the following recursive procedure:

(1) The complete graph $K_{2}$ with two vertices is primitive.

(2) Let $T$ be a primitive tree and $\{u, v\} \in E(T)$. Take two new vertices $x, y \notin$ $V(T)$. Then $T^{\prime}:=(V(T) \cup\{x, y\}, E(T) \cup\{\{u, x\},\{v, y\}\})$ is primitive. 
Note that $\# E(T)$ is odd for a primitive tree $T$.

Let $T$ be a tree. A set $\mathcal{D}=\left\{T_{1}, T_{2}, \ldots, T_{m}\right\}$ of subgraphs of $T$ is called a decomposition of $T$ with primitive trees if each $T_{i}$ is a primitive tree and $E(T)$ is the disjoint union of $E\left(T_{1}\right), E\left(T_{2}\right), \ldots, E\left(T_{m}\right)$. Note that $(\# E(T)+\# \mathcal{D}) / 2=$ $\sum_{i=1}^{m}\left(\# E\left(T_{i}\right)+1\right) / 2$ is an integer.

For $e \in E(T)$, let $T(e)$ denote the subgraph of $T$ with the vertex set $e$ and edge set $\{e\}$. Then $T(e)$ is a primitive tree and $\mathcal{D}_{0}:=\{T(e): e \in E(T)\}$ is a decomposition of $T$ with primitive trees. We call $\mathcal{D}_{0}$ the trivial decomposition of $T$ with primitive trees.

We set

$$
\begin{aligned}
m(T) & :=\min \{\# \mathcal{D}: \mathcal{D} \text { is a decomposition of } T \text { with primitive trees }\}, \\
b(T) & :=\frac{1}{2}(\# E(T)+m(T)) .
\end{aligned}
$$

We give a characterization of $b(T)$ in terms of a tree-like system of $T$.

Proposition 2.6. The integer $b(T)$ of a tree $T$ gives the minimum length among the tree-like systems of $T$.

Proof. We first show that there is a tree-like system of $T$ with length $b(T)$. For this purpose it is sufficient to prove that a primitive tree $T$ has a tree-like system of length $b(T)=(\# E(T)+1) / 2$. The proof proceeds by induction on \#E(T).

When $\# E(T)=1$, the only edge $e \in E(T)$ is a tree-like system of length $b(T)=1$.

We assume that $\# E(T) \geq 2$. By the definition of the primitive tree, there exist a primitive tree $T_{0}$, an edge $\{u, v\} \in E\left(T_{0}\right)$, and vertices $x, y \in V(T) \backslash V\left(T_{0}\right)$ such that

$$
T=\left(V\left(T_{0}\right) \cup\{x, y\}, E\left(T_{0}\right) \cup\{\{u, x\},\{v, y\}\}\right) .
$$

Since $\# E(T)=\# E\left(T_{0}\right)+2$, we have that $b(T)=b\left(T_{0}\right)+1$. Let $\mathcal{T}_{1}, \mathcal{T}_{2}, \ldots, \mathcal{T}_{r}$ be a tree-like system of $T_{0}$ of length $r:=b\left(T_{0}\right)$. We set $\mathcal{T}_{r+1}=\{\{u, x\},\{v, y\}\}$. Since $\{u, v\}$ is an edge of $T_{0}$ and there exists some $\mathcal{T}_{i}$ which contains $\{u, v\}$, the sequence $\mathcal{T}_{1}, \mathcal{T}_{2}, \ldots, \mathcal{T}_{r}, \mathcal{T}_{r+1}$ forms a tree-like system of $T$ with length $r+1=b(T)$.

Now we show that $b(T)$ gives the minimum length. Let $\Sigma: \mathcal{T}_{1}, \mathcal{T}_{2}, \ldots, \mathcal{T}_{r}$ be a tree-like system of $T$. If

$$
\Sigma^{\prime}: \mathcal{T}_{1}, \mathcal{T}_{2}, \ldots, \mathcal{T}_{i-1}, \mathcal{T}_{j}, \mathcal{T}_{i}, \mathcal{T}_{i+1}, \ldots, \mathcal{T}_{j-1}, \mathcal{T}_{j+1}, \ldots, \mathcal{T}_{r}
$$

is also a tree-like system of $T$ for a pair $i<j$ with $\# \mathcal{T}_{i}=1$ and $\# \mathcal{T}_{j}=2$, then we consider $\Sigma^{\prime}$ instead of $\Sigma$. If there is such a pair with respect to $\Sigma^{\prime}$, then we consider such a permutation for $\Sigma^{\prime}$, and we repeat this process as long as there is such a pair. Then from the beginning we may assume that $\Sigma$ does not allow such a permutation, since the length does not change. We rename the elements of $\Sigma$ as follows:

$$
\Sigma: \mathcal{T}_{1,1}, \mathcal{T}_{1,2}, \ldots, \mathcal{T}_{1, r_{1}}, \mathcal{T}_{2,1}, \mathcal{T}_{2,2}, \ldots, \mathcal{T}_{2, r_{2}}, \ldots, \mathcal{T}_{\ell, 1}, \mathcal{T}_{\ell, 2}, \ldots, \mathcal{T}_{\ell, r_{\ell}}
$$

such that $\# \mathcal{T}_{i, 1}=1, \# \mathcal{T}_{i, j}=2$ for $i=1,2, \ldots, \ell$ and $j>1$. We set

$$
\Sigma_{i}: \mathcal{T}_{i, 1}, \mathcal{T}_{i, 2}, \ldots, \mathcal{T}_{i, r_{i}}
$$

for $i=1,2, \ldots, \ell$. Let $T_{i}$ be the graph whose edge set consists of all edges appearing in $\Sigma_{i}$. Then $\Sigma_{i}$ is a tree-like system of $T_{i}$. It is easy to see that $T_{i}$ is a tree. We claim that $T_{i}$ is primitive. Let $T_{i, k}\left(k=1,2, \ldots, r_{i}\right)$ be the tree whose edge set is the union of $\mathcal{T}_{i, 1}, \mathcal{T}_{i, 2}, \ldots, \mathcal{T}_{i, k}$. We prove that $T_{i, k}$ is primitive for all $k$. Since 
$\# E\left(T_{i, 1}\right)=\# \mathcal{T}_{i, 1}=1$, the tree $T_{i, 1}$ is primitive. We assume that $T_{i, k}$ is a primitive tree. Set $\mathcal{T}_{i, k+1}=\{\{u, x\},\{v, y\}\}$ and assume that $\{u, v\} \in E\left(T_{i, k}\right)$. Since $T$ is a tree, $x$ and $y$ are distinct. Thus it is enough to prove that $x, y$ do not belong to $V\left(T_{i, k}\right)$. Suppose that $x$ belongs to $V\left(T_{i, k}\right)$. Then there exists a path in $T_{i, k}$ which connects $x$ and $u$. Since $\{x, u\} \in E(T) \backslash E\left(T_{i, k}\right)$, it follows that $T$ contains a cycle. This is a contradiction. Therefore $\mathcal{D}=\left\{T_{1}, T_{2}, \ldots, T_{\ell}\right\}$ is a decomposition of $T$ with primitive trees. Then the length of $\Sigma$ is equal to $r=(\# E(T)+\# \mathcal{D}) / 2$, which is greater than or equal to $b(T)$.

Example 2.7. Let $T$ be a tree with the vertex set $V(T)=\{u, v, x, y\}$ and with the edge set $\{\{u, v\},\{u, x\},\{v, y\}\}$ :

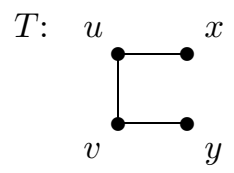

Then $T$ is a primitive tree. Indeed, it is obtained by one operation from a complete graph $T(\{u, v\}) \cong K_{2}$. In this case, $b(T)=2$ and $T$ has the tree-like system $\{\{u, v\}\},\{\{u, x\},\{v, y\}\}$, which is of length 2 .

\section{Proof of Theorem 1.1}

In this section we prove Theorem 1.1. In fact, we prove the following equality for a forest $G$ :

$$
\text { bight } I(G)=\operatorname{biara} I(G) \text {, }
$$

since we know

$$
\text { bight } I(G) \leq \operatorname{pd} K[V(G)] / I(G) \leq \operatorname{ara} I(G) \leq \operatorname{biara} I(G) .
$$

First we reformulate it in terms of graph theory. Let $G=(V(G), E(G))$ be a graph. A subset $C$ of the vertex set $V(G)$ is called a vertex cover of $G$ if it intersects all edges of $G$. If it is minimal under inclusion, it is said to be a minimal vertex cover. Since the correspondence from a minimal vertex cover $C$ of $G$ to the minimal prime ideal $P_{C}=\left(x_{v}: v \in C\right)$ of $I(G)$ is one-to-one, we have the following lemma:

Lemma 3.1. For a graph $G$ the big height bight $I(G)$ of the edge ideal $I(G)$ is equal to the maximum cardinality among the minimal vertex covers of $G$.

For Theorem 1.1 it is enough to consider the case of a tree. By Propositions 2.4 and 2.6 we have

$$
\text { bight } I(T) \leq \text { biara } I(T) \leq b(T) .
$$

Hence to show bight $I(T)=$ biara $I(T)$, it is enough to prove bight $I(T)=b(T)$. By Lemma 3.1 the equality bight $I(T)=b(T)$ is nothing but the following characterization of $b(T)$ in terms of graph theory.

Theorem 3.2. Let $T$ be a tree. Then $b(T)$ is equal to the maximum cardinality among the minimal vertex covers of $T$.

By (3.1) we know that bight $I(T) \leq b(T)$. Hence, by Lemma 3.1, to show the above theorem it is enough to prove the following lemma: 
Lemma 3.3. Let $T$ be a tree. Then there exists a minimal vertex cover $W$ of $T$ with $\# W \geq b(T)$.

Proof. The proof proceeds by induction on $n=\# V(T)$. When $n=2$, the tree $T$ is a complete graph with two vertices and we have $\# E(T)=m(T)=1$. Thus $b(T)=1$ and the assertion is trivially true.

We assume that the assertion is true for trees $T^{\prime}$ with $\# V\left(T^{\prime}\right)<n$. Let $T$ be a tree with $\# V(T)=n$. We denote by $l(T)$ the maximum length of paths in $T$.

If $l(T)=2$, then $T$ is a complete bipartite graph of type $(1, n-1): E(T)=$ $\left\{\left\{x_{0}, x_{1}\right\},\left\{x_{0}, x_{2}\right\}, \ldots,\left\{x_{0}, x_{n-1}\right\}\right\}$.
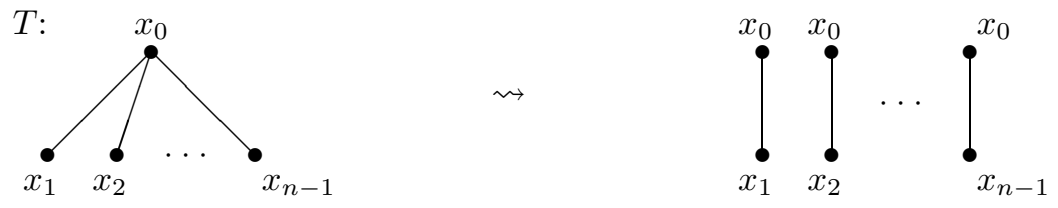

In this case $T$ has only the trivial decomposition of $T$ with primitive trees. Thus $\# E(T)=m(T)=n-1$ and $b(T)=n-1$. Then $W=\left\{x_{1}, x_{2}, \ldots, x_{n-1}\right\}$ is a minimal vertex cover of $T$ with $\# W=n-1=b(T)$.

When $l(T)=3$, the edge set of $T$ is

$$
E(T)=\left\{\left\{x_{0}, y_{0}\right\},\left\{x_{0}, x_{1}\right\},\left\{x_{0}, x_{2}\right\}, \ldots,\left\{x_{0}, x_{p}\right\},\left\{y_{0}, y_{1}\right\},\left\{y_{0}, y_{2}\right\}, \ldots,\left\{y_{0}, y_{q}\right\}\right\},
$$

where $p, q \geq 1$. We may assume that $p \geq q$. Then $\# E(T)=p+q+1$. Moreover $m(T)=1+p-q$.
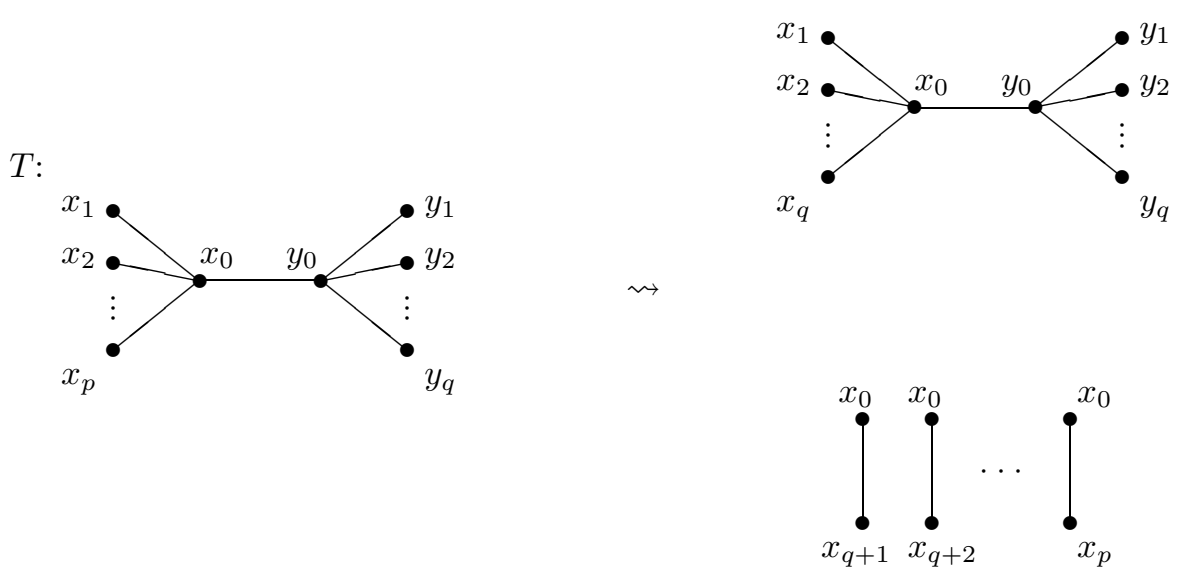

Hence

$$
b(T)=\frac{1}{2}((p+q+1)+(1+p-q))=p+1
$$

and $W=\left\{y_{0}, x_{1}, x_{2}, \ldots, x_{p}\right\}$ is a desired vertex cover of $T$.

Now we assume that $l:=l(T) \geq 4$. Let $\left\{v_{0}, v_{1}, \ldots, v_{l}\right\}$ be a path of $T$ with the maximum length. We divide the proof into two parts.

Case 1. First we assume that there exists a leaf $u$ of $T$ which is a neighbourhood of $v_{l-2}$. 


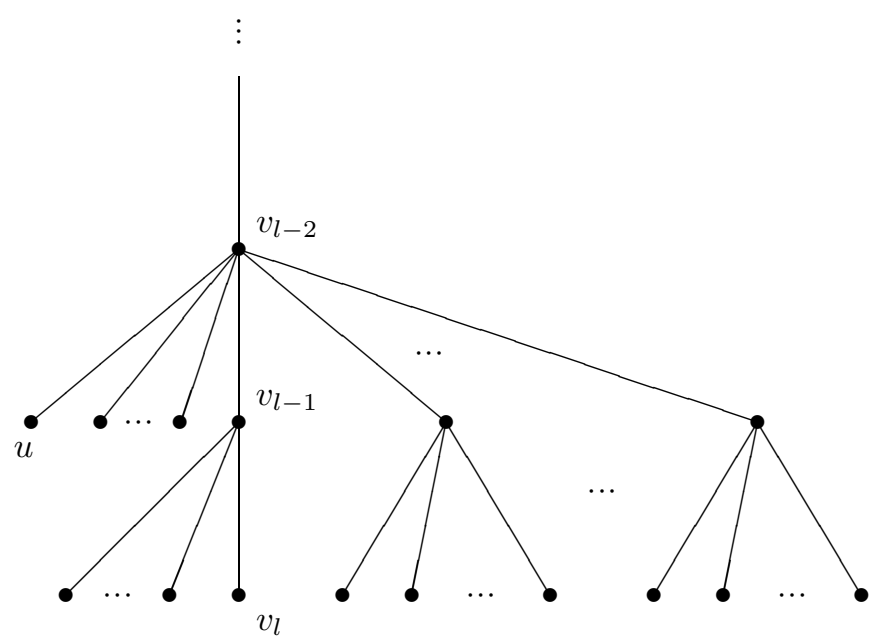

We consider the induced subgraph $T^{\prime}:=T_{V(T) \backslash\left\{u, v_{l}\right\}}$. Let $\mathcal{D}=\left\{T_{1}, T_{2}, \ldots, T_{m}\right\}$ be a decomposition of $T^{\prime}$ with primitive trees. We may assume that $\left\{v_{l-2}, v_{l-1}\right\} \in$ $E\left(T_{1}\right)$. Then the tree $\tilde{T}_{1}$ with the vertex set $V\left(T_{1}\right) \cup\left\{u, v_{l}\right\}$ and the edge set $E\left(T_{1}\right) \cup$ $\left\{\left\{v_{l-2}, u\right\},\left\{v_{l-1}, v_{l}\right\}\right\}$ is primitive. Thus $\tilde{\mathcal{D}}=\left\{\tilde{T}_{1}, T_{2}, \ldots, T_{m}\right\}$ is a decomposition of $T$ with primitive trees. Hence we have $b(T) \leq b\left(T^{\prime}\right)+1$.

By the inductive hypothesis, there exists a minimal vertex cover $W^{\prime}$ of $T^{\prime}$ with $\# W^{\prime} \geq b\left(T^{\prime}\right)$. If $v_{l-2} \notin W^{\prime}$, then $v_{l-1} \in W^{\prime}$. In this case, $W:=W^{\prime} \cup\{u\}$ is a minimal vertex cover of $T$ with

$$
b(T) \leq b\left(T^{\prime}\right)+1 \leq \# W^{\prime}+1=\# W .
$$

Next we assume that $v_{l-2} \in W^{\prime}$. If there is no leaf which is adjacent to $v_{l-1}$ in $T^{\prime}$, then $v_{l-1} \notin W^{\prime}$. Thus $W:=W^{\prime} \cup\left\{v_{l}\right\}$ is a minimal vertex cover of $T$ with

$$
b(T) \leq b\left(T^{\prime}\right)+1 \leq \# W^{\prime}+1=\# W .
$$

Otherwise, let $w_{1}, \ldots, w_{p}$ be all the leaves adjoining the $v_{l-1}$ in $T^{\prime}$. Then $W^{\prime \prime}:=$ $\left(W^{\prime} \backslash\left\{v_{l-1}\right\}\right) \cup\left\{w_{1}, \ldots, w_{p}\right\}$ is a minimal vertex cover of $T^{\prime}$ with $\# W^{\prime \prime} \geq \# W^{\prime}$. We set $W:=W^{\prime \prime} \cup\left\{v_{l}\right\}$. Then $W$ is a minimal vertex cover of $T$ with

$$
b(T) \leq b\left(T^{\prime}\right)+1 \leq \# W^{\prime \prime}+1=\# W .
$$

Case 2. Next we assume that there is no leaf adjoining $v_{l-2}$ in $T$. For $v \in V(T)$, we denote by $N(v)$ the set of neighbours of $v$. We set $U:=N\left(v_{l-2}\right) \backslash\left\{v_{l-3}\right\}:=$ $\left\{u_{1}, \ldots, u_{p}\right\}(p \geq 1), X_{i}:=N\left(u_{i}\right) \backslash\left\{v_{l-2}\right\}:=\left\{x_{i 1}, \ldots, x_{i q_{i}}\right\}\left(q_{i} \geq 1\right)$, and $X=$ $X_{1} \cup \cdots \cup X_{p}$. Note that $v_{l-1} \in U$ and $v_{l} \in X$. 


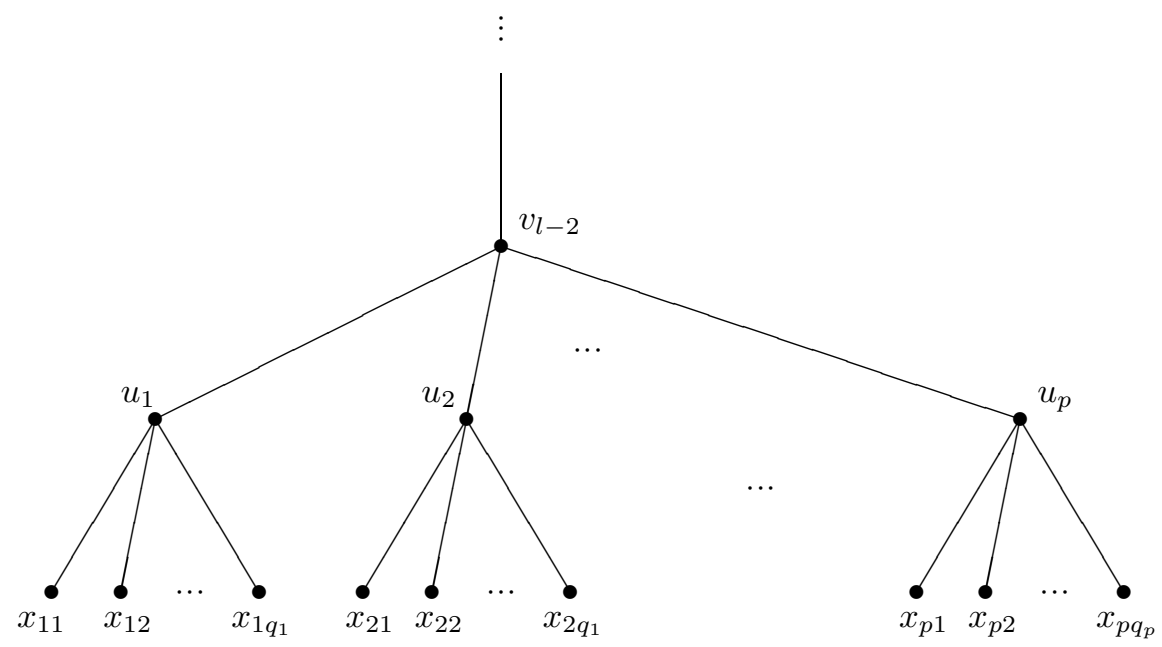

Also we set

$$
T^{\prime}:=T_{V(T) \backslash\left(\left\{v_{l-2}\right\} \cup U \cup X\right)}, \quad T^{\prime \prime}:=T_{\left\{v_{l-3}, v_{l-2}\right\} \cup U \cup X} .
$$

Then $T_{0}:=T_{\left\{v_{l-3}, v_{l-2}\right\} \cup U \cup\left\{x_{11}, x_{21}, \ldots, x_{p 1}\right\}}^{\prime \prime}$ is a primitive tree.

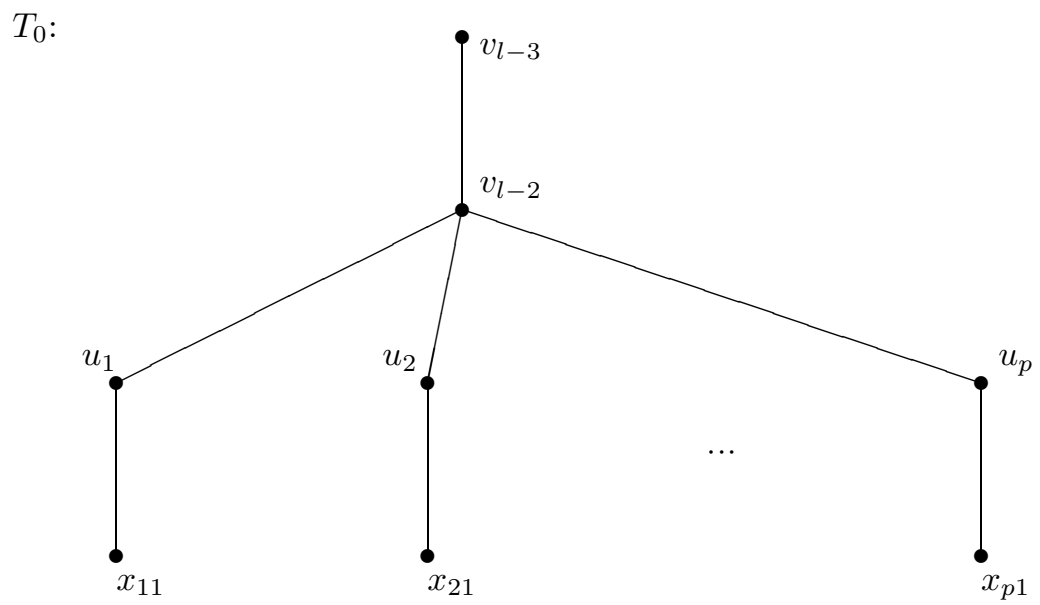

Indeed, we can construct $T_{0}$ inductively by adding edges with the following order:

$$
\begin{aligned}
& \left\{v_{l-2}, u_{1}\right\}, \\
& \left\{v_{l-2}, u_{2}\right\},\left\{u_{1}, x_{11}\right\}, \\
& \left\{v_{l-2}, u_{3}\right\},\left\{u_{2}, x_{21}\right\}, \\
& \cdots \\
& \left\{v_{l-2}, u_{p}\right\},\left\{u_{p-1}, x_{p-11}\right\}, \\
& \left\{v_{l-2}, v_{l-3}\right\},\left\{u_{p}, x_{p 1}\right\} .
\end{aligned}
$$


Thus $m\left(T^{\prime \prime}\right) \leq 1+\sum_{i=1}^{p}\left(q_{i}-1\right)$ and we have

$$
b\left(T^{\prime \prime}\right) \leq \frac{1}{2}\left\{\left(1+p+\sum_{i=1}^{p} q_{i}\right)+\left(1+\sum_{i=1}^{p}\left(q_{i}-1\right)\right)\right\}=1+\sum_{i=1}^{p} q_{i} .
$$

We set $W^{\prime \prime}=\left\{v_{l-2}\right\} \cup X$. Then $\# W^{\prime \prime}=1+\sum_{i=1}^{p} q_{i} \geq b\left(T^{\prime \prime}\right)$. By the inductive hypothesis on $T^{\prime}$, there exists a minimal vertex cover $W^{\prime}$ of $T^{\prime}$ with $\# W^{\prime} \geq b\left(T^{\prime}\right)$. Then $W^{\prime} \cap W^{\prime \prime}=\emptyset$ and $W:=W^{\prime} \cup W^{\prime \prime}$ is a minimal vertex cover of $T$ with

$$
\# W=\# W^{\prime}+\# W^{\prime \prime} \geq b\left(T^{\prime}\right)+b\left(T^{\prime \prime}\right) \geq b(T),
$$

as desired.

\section{REFERENCES}

[1] M. Barile, On the arithmetical rank of the edge ideals of forests, Comm. Algebra 36 (2008), 4678-4703. MR2473354 (2009j:13028)

[2] M. Barile, D. Kiani, F. Mohammadi and S. Yassemi, Arithmetical rank of the cyclic and bicyclic graphs, J. Algebra Appl. 11 (2012), no. 2, 1250039, 14 pp. MR2925452

[3] M. Barile and N. Terai, Arithmetical ranks of Stanley-Reisner ideals of simplicial complexes with a cone, Comm. Algebra 38 (2010), 3686-3698. MR2760684 (2011j:13033)

[4] M. Barile and N. Terai, The Stanley-Reisner ideals of polygons as set-theoretic complete intersections, Comm. Algebra 39 (2011), 621-633. MR 2773327

[5] V. Ene, O. Olteanu and N. Terai. Arithmetical rank of lexsegment edge ideals, Bull. Math. Soc. Sci. Math. Roumanie (N.S.) 53 (101) (2010), 315-327. MR2777678 (2011m:13037)

[6] J. He and A. Van Tuyl, Algebraic properties of the path ideals of a tree, Comm. Algebra 38 (2010), 1725-1742. MR2642022(2011e:13028)

[7] K. Kimura, Arithmetical rank of Cohen-Macaulay squarefree monomial ideals of height two, J. Commut. Algebra 3 (2011), 31-46. MR2782698

[8] K. Kimura, G. Rinaldo and N. Terai, Arithmetical rank of squarefree monomial ideals generated by five elements or with arithmetic degree four, to appear in Comm. Algebra.

[9] K. Kimura, N. Terai and K. Yoshida, Arithmetical rank of squarefree monomial ideals of small arithmetic degree, J. Algebraic Combin. 29 (2009), 389-404. MR2496313 (2009m:13030)

[10] K. Kimura, N. Terai and K. Yoshida, Arithmetical rank of monomial ideals of deviation two, in Combinatorial Aspects of Commutative Algebra (V. Ene and E. Miller, eds.), Contemporary Mathematics, 502, AMS (2009), 73-112. MR2583275 (2011c:13043)

[11] M. Kummini, Regularity, depth and arithmetic rank of bipartite edge ideals, J. Algebraic Combin. 30 (2009), 429-445. MR2563135 (2010j:13040)

[12] G. Lyubeznik, On the local cohomology modules $H_{\mathfrak{a}}^{\imath}(R)$ for ideals a generated by monomials in an R-sequence, in Complete Intersections, Acireale, 1983 (S. Greco and R. Strano, eds.), Lecture Notes in Mathematics, No. 1092, Springer-Verlag, 1984, pp. 214-220. MR775884 (86f:14002)

[13] M. Morales, Simplicial ideals, 2-linear ideals and arithmetical rank, J. Algebra 324 (2010), 3431-3456. MR2735392(2011j:13028)

[14] P. Mongelli, The arithmetical rank of a special class of monomial ideals, preprint, arXiv:1005.2586.

[15] T. Schmitt and W. Vogel, Note on set-theoretic intersections of subvarieties of projective space, Math. Ann. 245 (1979), 247-253. MR553343 (81a:14025)

[16] M. Varbaro, Symbolic powers and matroids, Proc. Amer. Math. Soc. 139 (2011), 2357-2366. MR.2784800

Department of Mathematics, Faculty of Science, Shizuoka University, 836 Ohya, Suruga-KU, SHIZUOKA 422-8529, JAPAN

E-mail address: skkimur@ipc.shizuoka.ac.jp

Department of Mathematics, Faculty of Culture and Education, Saga University, SAGA 840-8502, JAPAN

E-mail address: terai@cc.saga-u.ac.jp 